\title{
Vers une justification par les entreprises de leur appartenance au secteur de l'économie sociale et solidaire
}

\author{
Gurvan Branellec \\ Docteur en droit \\ Enseignant-chercheur ESC Bretagne-Brest \\ Chercheur associé UMR AMURE \\ France Business School Campus de Brest \\ 2, Av. de Provence \\ CS 23812 \\ 29238 Brest Cedex 3 \\ France
}

\begin{abstract}
Mots clefs : RSE, Economie sociale et solidaire, contrôle, efficacité, éthique.
Key Words: Corporate Social Responsibility (CSR), Social and solidarity-based economy, Control, Efficiency, Ethics.
\end{abstract}

Résumé : L'économie sociale et solidaire (ESS) fait partie du domaine de la réalité. Il est pourtant difficile de la définir, les entreprises de l'ESS utilisent des supports juridiques très différents. En l'absence de critère juridique, deux critères sont classiquement mis en œuvre pour délimiter les frontières de l'ESS : les finalités et le mode d'organisation interne (Lipietz, 2000, T. 1, p. 33). Les entreprises de l'économie sociale et solidaire vont donc se rattacher volontairement à l'ESS et vont chercher à obtenir une visibilité sur leurs différences par rapport aux entreprises capitalistes classiques. Ces entreprises devront mettre en place des mécanismes de gouvernance afin que leurs pratiques soient conformes à ces principes, elles vont se servir du droit comme d'un outil au service de cet objectif. La mise en œuvre d'un processus RSE va leur permettre de mesurer et de renforcer leur utilité sociale (1) ainsi que le caractère démocratique de leur fonctionnement (2).

\footnotetext{
Abstract: Social and solidarity-based economy (ESS) is part of the realm of reality. Since it is difficult to define, social and solidarity-based companies use completely different legal supports. Due to lack of the legal criterion, two criteria are habitually applied in order to determine the borders of the ESS: the purposes and the mode of internal organization (Lipietz, 2000, V.1, p.33). Therefore social and solidarity-oriented companies will voluntarily attach to the ESS and will try to draw attention to the difference they make when compared to classically capitalistic companies. These companies will have to implement certain mechanisms of management so that their practices can comply with these principles. They will make use of law as a tool to reach their goals. The enforcement of the CSR process will enable them to measure and strengthen their social usefulness (1) as well as the democratic character of their functioning (2).
} 


\section{Introduction}

L'économie sociale et solidaire (ci-après ESS) fait partie de la vie de millions de Français. Ceux-ci peuvent être impliqués directement dans une entreprise de l'ESS en tant qu'adhérents ou salariés ${ }^{1}$. Ils peuvent également être concernés indirectement par l'action de ces entreprises que ce soit en tant que donateur, bénéficiaire de l'action conduite, citoyen, consommateur...

L'ESS existe donc; elle est même un secteur fondamental de l'économie puisqu'il représente en France plus de $10 \%$ de l'emploi total. L'ESS pèserait entre 7 et $8 \%$ du PIB (Kaminski, 2009).

Pourtant malgré son existence celle-ci n'a pas de définition juridique précise et pleinement opératoire (Borgetto, 2006, p. 205).

Cela provient sans doute du fait qu'il est difficile d'identifier juridiquement les entreprises de l'ESS. En effet, il n'y a pas un mais de multiples supports juridiques possibles pour une entreprise de l'ESS. Il est possible d'y trouver aussi bien des formes civiles (association, mutuelle...) que commerciales (société à responsabilité limitée, société anonyme, société coopérative d'intérêt collectif (ci-après SCIC)...), à tel point que l'ESS est parfois qualifiée de « secteur multiforme $»^{2}$. La création d'un statut unique pour l'ensemble des entreprises de l'ESS a d'ailleurs été imaginée mais écartée. D'une part, le secteur de l'ESS ne semble pas en faire la demande, ce qui montre bien que ses acteurs trouvent des outils juridiques ad hoc au sein du corpus juridique (Lafore, 2003). D'autre part, il semble impossible d'arriver à mettre en place un modèle juridique uniforme qui pourrait servir de support à la fois à des ateliers d'insertion, des clubs sportifs, des régies de quartiers, des systèmes d'échange locaux ou un groupement de producteurs agricoles.

Ces différenciations entre les entreprises de l'ESS et les entreprises de l'économie classique ${ }^{3}$ ne peuvent pas non plus se faire selon les secteurs d'activité puisqu'on peut trouver ces dernières aussi bien dans les domaines marchands que non-marchands (distribution, service à la personne, transport...). Il n'est pas non plus possible de les identifier en droit par la mise en œuvre ou la création d'instruments juridiques particuliers (contrats, mode alternatif de règlement des litiges...) car les entreprises de 1'ESS utilisent des outils juridiques qui ne leur sont pas spécifiques (Borgetto, 2006, p. 210).

Ainsi, si les entreprises de l'ESS sont soumises au droit, le droit français ne met pas à leur disposition des outils spécifiques. Il est d'ailleurs assez révélateur de constater qu'il n'existe que très peu de littérature juridique consacrée à l'ESS (et encore celle-ci ne traite en général pas spécifiquement de l'aspect juridique, cf. par exemple Hurstel, 2009), les juristes s'intéressant d'abord aux structures juridiques. Il est ainsi très difficile lorsqu'on aborde l'ESS sous un angle juridique de se rattacher à un cadre théorique.

Pourtant ces entreprises se différencient de celles qui font partie de l'économie classique à tel point que l'on parle même de «tiers secteur ${ }^{4}$ ». Se pose donc la question de savoir comment définir une entreprise de l'ESS afin de savoir si une entreprise s'y rattache ou non.

Afin d'appréhender ce secteur, on part donc de principes fondateurs communs. L'ESS est ainsi définie comme le regroupement de structures économiques qui partagent des caractéristiques ${ }^{5}$ : primauté de la personne et de l'objet social, contrôle démocratique, adhésion volontaire, défense et mise en œuvre des principes de solidarité et de responsabilité, but non lucratif...

Deux critères sont classiquement mis en œuvre pour délimiter le secteur de l'ESS : les finalités et le mode d'organisation interne (Lipietz, 2000, T. 1, p. 33). Les entreprises doivent donc avoir une utilité sociale et une organisation interne démocratique pour entrer dans le champs de l'ESS. Ces deux

\footnotetext{
${ }^{1}$ L'économie sociale et solidaire employait en 2006 2,1 millions de salariés, soit 10\% de l'ensemble des salariés. Gaudron G., L'économie sociale emploie un salarié sur dix en 2006, INSEE.

${ }^{2}$ Rapport de la mission parlementaire sur le développement de l'économie sociale et solidaire, présidée par Francis Vercamer, remis au gouvernement le 28 avril 2010.

${ }^{3}$ Expression tirée du rapport précité.

${ }^{4}$ Notion développée dans un rapport de Bertrand Schwartz sur l'insertion sociale et professionnelle des jeunes paru en 1982.

${ }^{5}$ Charte européenne de l'économie sociale, Conférence européenne permanente des coopératives, mutualités, associations et fondations. Bruxelles 10 avril 2002. Charte de l'économie sociale, Comité national de liaison des activités mutualistes, coopératives et associatives. 1980.
} 
caractères fondant leur identité et leur rattachement au secteur de l'ESS. L'entrepreneuriat social et solidaire se fonde donc sur la seule affirmation subjective de valeurs et l'engagement sociétal qui en découle plutôt que sur le système de règles formalisées dans les différents statuts et lois (Parodi, 2010, p. 55).

Les entreprises françaises de l'ESS vont donc chercher à obtenir une visibilité sur ces différences par rapport aux entreprises capitalistes classiques. Ces entreprises devront ainsi mettre en place des mécanismes de gouvernance afin que leurs pratiques soient conformes à ces principes, elles vont se servir du droit comme d'un outil au service de cet objectif. Elles devront traduire juridiquement leur utilité sociale (1) ainsi que le mode démocratique de leur fonctionnement (2). Pour ce faire elles pourront mettre en œuvre un processus de responsabilité sociétale de l'entreprise (ci-après RSE) leur permettant de mesurer et de renforcer ces deux caractères de leur identité.

\section{Une appartenance à l'ESS par l'utilité sociale de l'entreprise}

Les entreprises de 1'ESS doivent avoir une utilité sociale. Pour ce faire, elles doivent prendre en considération l'environnement de l'entreprise ainsi que les relations que celle-ci noue (Freeman, 1984). Cette approche s'intéresse aux obligations des entreprises vis-à-vis des parties prenantes (stakeholders) qui sont « les individus et les groupements qui contribuent, volontairement ou non, à la capacité de créer de la valeur et de l'activité et qui en sont ses bénéficiaires potentiels et/ou en assument les risques » (Post, Preston, Sachs, 2002). Elle a donné lieu à la théorie des parties prenantes (stakeholder theory) qui identifie et organise les multiples obligations de l'entreprise envers les différents groupes qui y contribuent. Cette approche se pose donc comme « une alternative au modèle de gouvernance actionnariale et incite à une vision élargie des théories contractuelles des organisations (TCO) » (Gond et Mercier, 2006, p. 920).

Dans cette optique les entreprises de l'ESS vont avoir pour moteur de créer de la valeur sociale puisque le rattachement à l'ESS est volontaire (1.1). Pourtant, comme l'exprime M. Persais, « cette valeur ajoutée sociétale n'a de sens que démontrée et mesurée »(Persais, 2006, p. 22) (1.2).

\subsection{D'une utilité sociale voulue}

Les acteurs de l'ESS sont avant tout des entreprises. Etymologiquement le terme entreprendre signifie attaquer (1140) : il a une connotation offensive. En 1559, le terme prend la signification « conquérir par la flatterie, essayer de séduire ». Nous sommes donc bien loin du côté social et solidaire. La définition moderne de l'entreprise se confond avec celle de la société au sens juridique (article 1832 du Code civil). La société est un contrat conclu pour affecter à une entreprise commune des biens ou leur industrie en vue de partager le bénéfice ou de profiter de l'économie qui pourra en résulter. Nous sommes donc face à des personnes qui organisent leur coopération en mutualisant leurs ressources et en associant leurs compétences. Il s'agit d'un entrepreneuriat collectif. Les règles classiques de l'ESS excluent de facto le cas de l'entrepreneuriat individuel (libre adhésion, égalité des membres, double qualité...). Jusque-là il n'y a pas de critère de distinction des entreprises de l'ESS par rapport aux autres.

La recherche du lucre ou, pour être plus exact, la recherche ou non d'une répartition des bénéfices semble permettre de les distinguer. Les entreprises de l'ESS ont en commun le fait que la recherche du profit n'est pas l'objectif essentiel de leur action (Autès, 2006, p. 94). Jusqu'à la fin du XIXème siècle, les organisations qui n'avaient pas d'objectif lucratif devaient «s'ajuster au modèle de la société, ou n'exister que de fait »(Castro, 1996, p. 53), progressivement le législateur a ensuite institué des organisations à but non-lucratif (associations, fondations...). Toutefois, dans tous les cas, les entreprises de l'ESS vont rechercher l'efficacité de leur action. Lorsqu'elles s'inscrivent dans une logique de marché, elles rechercheront la viabilité économique de leur activité. Lorsqu'elles visent la réalisation d'un profit, celui-ci va être un moyen d'accomplir une action. L'action de ces entreprises va tendre à l'utilité sociale. L'entrepreneur social est ainsi défini par la School for Social Entrepreneurs comme «celui qui opère de manière entrepreneuriale mais pour le bien public ou d'une collectivité plutôt que pour s'enrichir ». 
L'utilité sociale est « une notion dérivée de celle de l'intérêt général» (Euillet, 2002, p. 210). La notion d'intérêt général illustre la prise en compte actuelle de l'extérieur par les entreprises de l'ESS (Hiez et Laurent, 2011, p. 36). La notion d'intérêt général, "épine dorsale du droit public » (Walline, 1999, p. 277), va progressivement céder le pas à celle d'utilité sociale créée au profit de catégories mouvantes et mal définies d'usagers (Parodi, 2010, p. 43) et qui va reposer sur " un choix de valeur » (Sue, 1994). Il en résulte que cette notion d'utilité sociale n'est pas une notion juridique ; elle est malaisée à définir car protéiforme et contingente. L'utilité sociale peut se manifester ou se cristalliser à quatre niveaux : un premier dans lequel elle rejoindra l'utilité individuelle, un second dans lequel elle sera une utilité collective, à un troisième niveau l'utilité sera sociale externe et à un dernier niveau l'utilité sera sociétale (halo sociétal) (Parodi, 2010, p. 44-46). Ces différents niveaux montrent bien que l'utilité sociale ne saurait être réduite à l'utilité individuelle ou collective mais qu'elle « déborde de toute part dans les cercles concentriques du halo sociétal ou les composantes de la société » (Parodi, 2010, p. 54). Cette ouverture sur l'extérieur rejoint l'idée que l'entreprise solidaire et sociale a une responsabilité et une finalité sociales. Les entreprises de l'ESS ont ainsi un devoir envers la collectivité à laquelle elles empruntent des ressources pour leur financement. Cette démarche responsable rejoint la montée en puissance du concept de responsabilité sociale de l'entreprise : celleci devant être « citoyenne».

En effet, le comportement d'une entreprise n'est pas uniquement susceptible d'affecter les créateurs ou dirigeants, il peut également concerner d'autres parties prenantes internes (adhérents, salariés...) ou externes (consommateurs, créanciers, clients, citoyens...). Le concept de responsabilité sociale de l'entreprise (RSE) permet de faire entrer les parties prenantes dans la gouvernance d'entreprise grâce aux valeurs sociales, environnementales et éthiques. Cette prise en compte par les entreprises d'une responsabilité sociétale est assez ancienne (mouvement coopératif ou mutualiste, mécénat, politique sociale de grandes entreprises) mais elle se développe aujourd'hui à cause des pressions sociales, environnementales et économiques (Desbarats, 2008, p. 68). La société civile amène les entreprises à adopter des démarches de responsabilité sociale. Il peut s'agir d'une démarche sincère d'entreprises soucieuses de considérations éthiques, ou d'un souhait d'éviter des mises en jeu de leur responsabilité voire encore d'une stratégie marketing visant à améliorer leur image de marque (une entreprise capitaliste peut souhaiter améliorer son image de marque en créant une filiale qui serait une entreprise sociale et solidaire). Cette prise en compte par les entreprises de leur responsabilité sociale se caractérise surtout par des démarches volontaires.

Les entreprises se dotent de chartes sociales, codes de conduite, codes de déontologie, référentiels d'engagement... Il s'agit de «signes de qualités privés » (Blin-Franchomme, 2009, p. 72) qui se développent en dehors de la contrainte de la loi. La Commission européenne met l'accent sur cette autorégulation des entreprises en définissant la RSE comme «l'intégration volontaire des préoccupations sociales et écologiques des entreprises à leurs activités commerciales et leurs relations avec les parties prenantes ${ }^{6}$ et a précisé en 2011 que «la responsabilité sociale des entreprises concerne les actions de celles-ci qui vont au-delà des obligations juridiques qui leur incombent à l'égard de la société et de l'environnement $»^{7}$.

Ces initiatives privées, constitutives d'un droit souple (soft law), se rencontrent dans des domaines variés : accords environnementaux (éco-labels, réduction des emballages...), engagements sociaux (développement durable, commerce équitable...), règles de conduite financière ou éthique (rémunération des dirigeants, non-discrimination, amélioration des conditions de travail des salariés)... Les domaines concernés, ainsi que les valeurs consacrées, sont d'une grande diversité. La forme de ces engagements et les formules employées le sont tout autant. Ces démarches volontaires peuvent aller de formules floues qui comportent des engagements imprécis jusqu'à des adjonctions aux règlements internes des entreprises (Pereira, 2009, p. 30) ou des engagements pris par des entreprises ou branches sectorielles qui vont produire des conséquences juridiques. Ces chartes et codes de conduite permettent aux entreprises de formaliser et de communiquer sur une démarche responsable.

\footnotetext{
${ }^{6}$ Livre vert, juillet 2001.

${ }^{7}$ Responsabilité sociale des entreprises: une nouvelle stratégie de l'UE pour la période 2011-2014, Communication de la Commission au Parlement européen, au Conseil, au Comité économique et social européen et au Comité des régions. Com (2011), 681.
} 
Ainsi, il apparaît que toutes les entreprises doivent prendre en compte leurs impacts sociaux, sociétaux et environnementaux. Les entreprises de l'ESS doivent donc justifier de plus en plus de leur utilité sociale puisque les entreprises capitalistiques communiquent de plus en plus sur des finalités sociales ou en tout cas une prise en considération des questions sociales. Les entreprises de l'ESS ont donc tout intérêt à développer une démarche permettant «d'apprécier l'adéquation des comportements de l'organisation au regard des valeurs de l'économie sociale, voire de valoriser ce qui fait les spécificités du secteur » (Persais, 2006, p. 23).

\section{2 ...A une utilité sociale mesurée et justifiée}

La prise en compte par les entreprises capitalistes des questions de RSE et la multiplication des démarches RSE initiées font craindre que celles-ci ne soient que des artéfacts publicitaires, «que le quantitatif ou le marketing prime sur la qualité » (Delga, 2005, p. 8). Il y a dès lors un risque de perte de lisibilité entre les entreprises de l'ESS et celles capitalistes.

En outre, au-delà de ce risque de perte d'identité, la plupart des entreprises de l'ESS perçoivent un soutien ou un financement, tant de leurs membres que des institutions publiques (Etat ou collectivité territoriale), en fonction de l'utilité sociale que ceux-ci leurs reconnaissent. Elles ont donc tout intérêt à pouvoir justifier de cette utilité. Plus spécifiquement, cette utilité sociale peut également permettre aux associations d'échapper à l'imposition commerciale. En effet, l'administration fiscale réalise un raisonnement en étapes afin de déterminer si une association qui développe des activités lucratives relève de l'imposition commerciale. Elle prend notamment en considération au cours d'une de ces étapes le produit proposé et le public visé. La justification d'une utilité sociale, s'agissant de ces deux critères, permettra de bénéficier d'une exonération de l'imposition commerciale (Hély, 2010, p. 213).

Les enjeux financiers et politiques doivent donc pousser les entreprises de l'ESS à mesurer et à pouvoir justifier de leur utilité sociale. Le principal problème qui se pose est que l'utilité sociale est une notion extra-financière et donc difficilement quantifiable. Pourtant, la lisibilité de cette utilité est le gage d'une bonne comparabilité et d'une meilleure gouvernance des entreprises de l'ESS.

Cette démarche d'objectivisation de l'utilité sociale a été entreprise dès les années 2000 ; Jean Gadrey a notamment cherché à examiner les outils qui permettraient de passer de convictions affichées par les acteurs en matière d'utilité sociale, convictions valides mais peu étayées, à des "évaluations socioéconomiques » plus fiables, et donc plus convaincantes dans le débat public (Gadrey, 2006, p. 237 et s.). Des économistes se sont également engagés dans cette voie grâce aux programmes de recherche de la Délégation interministérielle à l'économie sociale (DIES) et de la Mire (mission recherche du Ministère de l'emploi, du travail et de la cohésion sociale) (Parodi, 2010, p. 43). Il n'existe toutefois pas d'outils standards, les outils existants sont «artisanaux» (Parodi, 2010, p. 52). Il s'agit d'outils élaborés par des équipes mixtes (acteurs-chercheurs-accompagnateurs) que les entreprises de l'ESS vont pouvoir reprendre afin de les adapter à leur projet ou à leurs spécificités.

Pour ce faire, l'entreprise de l'ESS va devoir ouvrir un dialogue avec ses parties prenantes afin d'identifier des critères et des indicateurs objectifs. S'agissant de l'identification de ces parties prenantes, l'entreprise de l'ESS doit développer une approche pragmatique. Elle doit identifier "les acteurs significatifs pour l'organisation », ceux qui seront « représentatifs d'intérêts " pertinents » au regard des impacts générés » (Persais, 2006, p. 27). Les différentes parties prenantes pourront avoir des attentes différentes et parfois contradictoires en ce qui concerne l'entreprise. Celle-ci pourra donc être amenée à réaliser des choix parmi les objectifs qui lui sont assignés. Pour ce faire, l'entreprise devra «éclairer des possibilités de compromis et d'arbitrage entre différentes catégories d'acteurs » (Lapenu et Doligez, 2007, p. 60).

La première étape sera donc de définir quelle est l'utilité sociale de l'entreprise. L'utilité sociale fait l'objet de plusieurs définitions abstraites. Cette notion est présente en droit notamment par le biais de la définition des SCIC qui ont pour objectif « la production ou la fourniture de biens et de services d'intérêt collectif, qui présentent un caractère d'utilité sociale» (Amblard, 2010, p. 26). L'administration fiscale considère qu'est d'utilité sociale «toute réponse à un besoin qui n'est pris en charge ni par l'Etat, ni par le marché » (Hély, 2010, p. 208). Le Conseil National de la Vie Associative a également élaboré une définition de l'utilité sociale qui concernerait une "activité qui tend à satisfaire un besoin qui n'est pas pris en compte par le marché ou qui l'est de façon insatisfaisante ». Ces définitions sont toutefois beaucoup trop larges pour être opérantes (paradoxalement les tribunaux 
se réfèrent de plus en plus souvent à cette notion (Amblard, 2010, p. 32)). Il va appartenir à chaque entreprise de l'ESS de clarifier ses finalités afin de pouvoir élaborer un projet sociétal avec les parties prenantes. Pour ce faire elle devra être attentive à impliquer toutes les parties prenantes car l'utilité sociale est tant interne qu'externe. Pour renforcer son impact social l'institution va devoir « clarifier sa mission et s'assurer que ses actions sont en cohérence avec sa mission » (Lapenu et Doligez, 2007, p. 48). L'entreprise va devoir également élaborer un diagnostic de la situation qui lui permettra d'avoir une vision claire de la situation présente afin qu'elle puisse améliorer la plus-value sociale apportée par l'entreprise.

Des outils de mesure de l'impact social d'une action et donc in fine de l'utilité sociale d'une entreprise mettant en œuvre ces actions existent ou sont en cours de formalisation. L'Agence de Valorisation des Initiatives Socio-Economiques ainsi que des fondations d'entreprise membres du réseau européen European Venture Philanthropy Association (aidés par un cabinet de consultant néerlandais) ont élaboré un indicateur «Social E-valuator» qui permet de calculer le Social Return On Investment sur la base d'indicateurs quantitatifs (Hély, 2010, p. 214) développés par les entreprises de l'ESS.

Il existe donc des outils permettant à une entreprise de mesurer son utilité sociale.

Outre ces démarches volontaires, l'Etat peut aussi fournir des outils permettant aux entreprises de l'ESS de bénéficier d'une plus grande transparence en ce qui concerne leur utilité sociale.

Ainsi s'agissant du commerce équitable, les pouvoirs publics sont intervenus pour donner plus de lisibilité à ce secteur. En effet, les consommateurs de produits équitables sont confrontés à une multitude d'offres provenant soit de filières intégrées soit de filières labélisées. Pour ces dernières, la labellisation est le fait de prestataires de services qui vont attester qu'un produit ou une entreprise est conforme à un cahier des charges. Afin de garantir au consommateur une plus grande lisibilité et fiabilité de ces labels, le décret n ${ }^{\circ}$ 2007-986 du 15 mai 2007 prévoit une procédure de reconnaissance

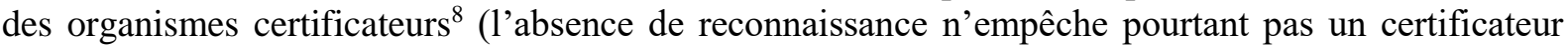
d'intervenir sur le marché....). L'Etat propose également un agrément d' « entreprise solidaire » dont le décret $\mathrm{n}^{\circ}$ 2009-304 du 18 mars 2009 pose les conditions de reconnaissance (la loi $\mathrm{n}^{\circ} 2012-387$ du 22 mars 2012 a supprimé l'agrément préfectoral de la SCIC qui servait notamment à apprécier l'utilité sociale du projet).

Il apparaît donc que les entreprises de l'ESS devront, dans une démarche pragmatique, adopter ou élaborer des outils leur permettant de justifier que l'organisation se donne les moyens de ses objectifs sociaux. Ces outils devront être adaptés à la taille de l'organisation, à son histoire, à ses modes de fonctionnement, au contexte dans lequel elle évolue...

Une démarche d'évaluation de l'utilité sociale d'une entreprise s'inscrit dans un cadre plus large qui est celui de sa performance sociale. La performance sociale dépendra également de la gouvernance et de la dynamique interne de l'entreprise. Il s'agit du second critère de distinction des entreprises de l'ESS.

\section{Une appartenance à l'ESS par le mode d'organisation interne de l'entreprise}

L'entreprise sociale et solidaire se caractérise par son mode d'organisation interne. Il y aurait en effet un caractère démocratique et multipartenarial de sa direction.

L'intégration de ces caractères va dépendre pour beaucoup de la forme juridique de l'entreprise de l'ESS. Les supports juridiques classiques de l'ESS (association, coopérative...) semblent favoriser le respect des intérêts des parties prenantes internes (2.1). Pourtant, ces formes juridiques ne constituent pas un gage du caractère démocratique de l'organisation interne, l'entreprise de l'ESS devra donc développer cette démocratie et formaliser des modes de gouvernance lui permettant d'aboutir à une gestion responsable (2.2).

\subsection{D'une organisation interne traditionnellement démocratique}

\footnotetext{
${ }^{8}$ Six critères doivent être respectés : amélioration des conditions de vie des producteurs défavorisés, indépendance, transparence, présence auprès des producteurs défavorisés, contrôles auprès des importateurs, accompagnement et sensibilisation.
} 
Les entreprises qui sont le support traditionnel de l'ESS (association, fondation, coopérative et mutuelle) vont avoir pour préoccupation la préservation du capital humain. L'économie devra être au service de l'homme et ce notamment par la démocratisation de la gestion. Ces entreprises paraissent donc être un lieu privilégié pour l'existence d'une réelle démocratie interne (Neyret, 2006, p. 38). En effet, «les processus de décision au sein de ces organisations sont fondés sur des principes et procédures démocratiques qui en principe garantissent la participation de leurs membres aux prises de décision» (Enjolras, 2005, p. 57). Cette gestion démocratique est classiquement désignée sous le vocable de gouvernance d'entreprise. La notion de gouvernance d'entreprise fait référence à l'ensemble des organes et règles de décision, d'information et de surveillance permettant aux ayants droit et partenaires d'une institution, de voir leurs intérêts respectés et leurs voix entendues dans le fonctionnement de celle-ci. Il est possible de reprendre la définition de la gouvernance donnée par le Professeur Chaput qui serait «l'art de gouverner en recherchant une solution impartiale aux conflits d'intérêts » (Chaput, 2005, p. 25-26).

Les structures de la gouvernance des entreprises de l'ESS dépendront d'abord de leur forme juridique. En effet, si «l'habit juridique ne suffit pas à faire le bon moine de l'entreprise de l'économie sociale, il a le mérite essentiel d'en fixer la règle » (Parodi, 2010, p. 55).

S'agissant de l'association, cette forme juridique se prête tout particulièrement aux pratiques de bonne gouvernance car les associations fonctionnent souvent sur le principe « un homme, une voix ${ }^{9}$ et, si les statuts de l'association peuvent déroger à ce principe, la jurisprudence interprète strictement les clauses qui portent atteinte à l'égalité des membres de l'association.

La mutuelle est également une forme juridique propice à une bonne gouvernance interne puisque le système de direction de celle-ci repose également sur la règle «un homme, une voix $»^{10}$. Un auteur considère ainsi que le Code de la mutualité «est d'abord et même essentiellement un code de gouvernance » (Lenoir, 2009, p. 397). Les mutuelles ont d'ailleurs souhaité rendre plus effective et plus visible la gouvernance mutualiste en développant des recommandations et des propositions (Herry et Lenoir (Dir.), 2006).

La fondation, qui permet d'affecter des biens à la réalisation d'un intérêt général, doit être indépendante de ses fondateurs. Deux formules sont possibles pour sa gouvernance (pour celles reconnues d'utilité publique). La première formule est classique, il s'agit de la direction par un conseil d'administration. La seconde est offerte par les statuts types du 2 avril 2003. Cette formule va dissocier la direction de la fondation entre un conseil de surveillance qui aura en charge la politique et la stratégie de la fondation et un directoire qui aura un rôle opérationnel. Cette seconde formule favorise une bonne gouvernance de la fondation même si elle peut être lourde à mettre en place pour de petites fondations.

La forme juridique de la coopérative et les règles de gouvernance interne de celles-ci permettent également une gestion qui prendra en considération les intérêts des diverses parties prenantes. Cette bonne gestion est ainsi favorisée grâce à deux grands principes coopératifs qui sont la double-qualité et la gestion démocratique. La double-qualité signifie que les associés sont également des coopérateurs (clients, fournisseurs, salariés...). La coopérative a été déclinée en des formes spécifiques (société coopérative européenne, société coopérative ouvrière de production (ci-après SCOP)...), la dernière grande modernisation de cette forme juridique étant la création de la SCIC par la loi du 17 juillet 2001. Celle-ci a été qualifiée par un auteur d' "outil de développement des projets d'économie sociale et solidaire dans le cadre d'une politique d'insertion par l'activité économique » (Hérail, 2002, $\mathrm{n}^{\circ} 47$ et 425). Les SCIC se caractérisent par un aménagement de la règle de la double qualité puisqu'elle admet jusqu'à 5 catégories d'associés qui peuvent en faire partie (la SCIC devra au moins comprendre trois sortes d'associés dont les salariés et les bénéficiaires font obligatoirement partie). Elles se caractérisent également par une atténuation du principe classique au sein des coopératives « un homme, une voix ».

\footnotetext{
${ }^{9}$ Cette règle permet d'avoir une stricte gestion démocratique puisqu'on ne va pas tenir compte du montant du capital apporté, chaque personne physique ayant une voix (Plantamp, 2005, p. 465).

${ }^{10}$ Le caractère démocratique est également renforcé par la solidarité qui existe entre ses membres puisque l'article L.111-1 du Code de la mutualité prévoit que les mutualités mènent « notamment au moyen de cotisations versées par leurs membres, une action de prévoyance, de solidarité, d'entraide, dans les conditions prévues par leurs statuts, afin de contribuer au développement culturel, moral, intellectuel et physique de leurs membres et à l'amélioration de leurs conditions de vie ».
} 
Une SCIC, comme d'autres sociétés coopératives, (coopératives agricoles, SCOP, union de coopératives artisanales ou maritimes), peut instituer un système non paritaire de répartition du pouvoir $^{11}$. On voit donc une atténuation au principe de gestion démocratique. Cette atténuation n'est pas spécifique aux SCIC.

Ainsi s'agissant des SCOP, des chercheurs de l'Equipe de Socio-Economie Associative et Coopérative (ESEAC) de Grenoble ont identifié quatre types d'orientation des activités parmi les SCOP enquêtées. Ceux-ci vont d'une logique coopérative à forte démocratie participative à une logique financière qui est traditionnellement considérée comme peu propice à une bonne gouvernance de l'entreprise (Neyret, 2006, p. 36).

En ce qui concerne les associations, des exemples non anecdotiques de tensions ont mis en lumière une contradiction entre les valeurs affichées et les pratiques internes, notamment en ce qui concerne la gestion du personnel. Ainsi, l'association Emmaüs a connu un important conflit avec les syndicats de salariés Confédération Générale du Travail (CGT), Force Ouvrière (FO) et Solidaires Unitaires Démocratiques (SUD) qui a abouti à une grève des salariés qui souhaitaient une amélioration des conditions de travail (Hély, 2010, p. 206). Il peut être également parfois difficile de trouver des spécificités de l'ESS dans leur façon de penser et de conduire les relations professionnelles ( négociation avec des fournisseurs ou sous-traitants...) (Neyret, 2006, p. 38).

Il semble donc que les formes juridiques traditionnellement dévolues à l'ESS, si elles favorisent des pratiques de bonne gouvernance, ne permettent pas de garantir que celles-ci existeront forcément. Il est donc intéressant d'étudier à présent les outils que les entreprises de l'ESS peuvent développer pour renforcer et mesurer le caractère démocratique de leur gouvernance.

\section{2 ...A un caractère démocratique renforcé et mesuré}

Le caractère démocratique du fonctionnement de l'entreprise est une notion difficile à définir puisqu'elle recouvre en fait une multitude d'objectifs. Il y aura bien sûr la question de savoir comment est pilotée l'entreprise, si celle-ci intègre suffisamment les dimensions éthiques dans son processus décisionnel. Il y aura également la question de savoir comment sont représentées les différentes parties prenantes, comment sont répartis les éventuels bénéfices tirés de l'activité, si l'entreprise prend en considération les questions de diversité, de mixité sociale... Le réseau Emes retient trois indicateurs de structure de gouvernance qui caractériseraient une entreprise de l'ESS : un degré élevé d'autonomie, un pouvoir de décision non basé sur la détention de capital et une dynamique participative impliquant différentes parties concernées par l'activité (Defourny et Nyssens, 2011, p. 24). Ces indicateurs ne sont toutefois pas normatifs, il s'agit de critères abstraits qui peuvent servir de boussole aux entreprises de l'ESS. En effet, le renforcement et la mesure du caractère démocratique vont dépendre de chaque entreprise. Celle-ci devra veiller à mettre en place un dialogue social en interne qui permette à l'entreprise, en fonction de sa structure et de ses valeurs, de renforcer la démocratie interne en équilibrant les différents intérêts en jeu. Il y aura déjà une distinction importante à faire entre les entreprises de l'ESS qui emploient des salariés et celles qui n'en emploient pas. Lorsqu'elles emploient des salariés, elles pourront intensifier le caractère démocratique de leur gouvernance en associant ceux-ci étroitement aux décisions prises. Elles pourront également développer des normes ambitieuses dans la formation, les conditions de travail ou les relations entre la direction et le personnel (Persais, 2006, p. 20).

S'agissant de la direction des entreprises de l'ESS, contrairement à l'économie traditionnelle, les dirigeants ne supportent pas les conséquences de leurs décisions en termes de conséquences financières. Or, les structures de gouvernement sont définies «par la nature des incitations qu'elles

\footnotetext{
${ }^{11}$ Un vote par collège représentatif de chaque composante de la SCIC (salariés, bénéficiaires, bénévoles, collectivités publiques et contributeurs) peut être organisé. Chaque individu va disposer d'une voix dans son collège et chaque collège possédera un nombre de voix égal à l'assemblée générale. Ce système peut être pondéré puisque les statuts peuvent définir le nombre de délégués qui représenteront chaque collège à l'assemblée générale et le nombre de voix attribué à ces délégués sera fonction de l'effectif des associés ou de la qualité des engagements de ceux-ci dans la coopérative. La part de chaque collège ne peut dépasser $50 \%$ ni devenir inférieure à $10 \%$ des droits de vote.
} 
incorporent et auxquelles les dirigeants répondent »(Enjolras, 2005, p. 64). L'entreprise de l'ESS devra donc être particulièrement attentive aux incitations axiologiques. C'est vrai pour les dirigeants mais également pour les cadres ou simples salariés qui travaillent dans les entreprises de l'ESS. Les études statistiques montrent l'existence d'un différentiel important entre les rémunérations au sein des entreprises de l'ESS et celles au sein des entreprises capitalistes (Le Gros et Narcy, 2004, p. 215), il sera donc important que l'activité soit source d' «externalités positives » (Hély, 2010, p. 211). L'utilité sociale permettra à l'entreprise de l'ESS d'avoir une meilleure gouvernance interne. Réciproquement, la structure de la gouvernance «peut être considérée comme un ensemble de caractéristiques organisationnelles destinées à garantir la poursuite de la mission sociale » (Defourny et Nyssens, 2011, p. 29). Leur structure de gouvernance (finalités formelles, propriété, distribution des droits de propriété, procédures de prise de décision) doit être adaptée aux exigences de l'action collective et doit permettre la mutualisation des ressources et la production d'un capital de confiance (Enjolras, 2009, p. 64).

Les entreprises devront également accepter de rendre compte de leurs actes aux parties prenantes.

Là encore on trouvera la nécessité pour l'entreprise d'identifier ces parties prenantes, de mettre en place des instances de dialogue et de concertation avec celles-ci. Cette gestion démocratique est parfois difficile à mettre en place car les entreprise de l'ESS peuvent être confrontées à une multitude de parties prenantes: les dirigeants, le conseil d'administration, les salariés, les bénévoles, les donateurs, les collectivités territoriales, l'Etat, voire même les bénéficiaires de l'action conduite... A partir de là, l'entreprise va devoir créer les critères d'une gestion efficace, conforme à l'intérêt social défini mais qui permette également la prise en compte des intérêts des parties prenantes de l'organisation. Ces parties prenantes peuvent participer à la vie quotidienne de l'entreprise en étant impliquées dans des instances formelles (assemblées générales) ou non formelles (comités d'usagers) (Defourny et Nyssens, 2011, p. 28). Les intérêts d'une bonne gouvernance d'une entreprise de l'ESS sont multiples. Il a d'abord été expliqué que ces entreprises tendaient à l'utilité sociale, elles doivent donc être irréprochables quant à leur gestion interne, ce qui leur permettra de bénéficier d'un capital confiance qui est fondamental ne serait-ce que pour collecter des fonds, obtenir l'investissement de ses membres (associés, salariés, bénévoles...) ou accroitre dans le temps le capital de confiance de l'organisation et sa capacité à faire face aux échecs de coordination (Enjolras, 2009, p. 79). Une bonne gouvernance permettra en outre de clarifier les fonctions ainsi que la responsabilité de chacun dans le fonctionnement de l'entreprise. Cette bonne gestion interne permettra logiquement de « diffuser ses effets à l'extérieur de la structure et favoriser le développement de comportements participatifs, citoyens dans les étagements des espaces publics emboîtés » (Parodi, 2010, p. 47).

Si les impératifs de bonne gouvernance s'appliquent à toutes les entreprises, en revanche les pratiques de cette gouvernance devront être adaptées en fonction de la taille, de la nature et de l'objet de l'organisation ${ }^{12}$.

L'instauration d'une gouvernance démocratique suppose qu'elle soit éclairée. Cela nécessite l'établissement d'un bilan suffisamment précis qui pourra constituer le départ d'un dialogue avec les différents acteurs de l'entreprise. A ce titre les entreprises de l'ESS pourront réaliser un bilan social qui est un véritable outil de gouvernance permettant à l'entreprise d' "informer sur la situation de l'organisation dans des catégories non référencés dans les bilans comptables ou sociaux conventionnels » (Persais, 2006, p. 14). Le Centre des jeunes dirigeants et des acteurs de l'économie sociale a d'ailleurs élaboré un bilan sociétal adapté aux entreprises de l'ESS et qui permet d'obtenir un outil participatif d'évaluation en vue de l'amélioration des pratiques.

L'objectif final est d'aboutir à ce que l'entreprise s'interroge de façon continue sur les pratiques mises en œuvre et sur les voies d'améliorations de celles-ci.

\section{Conclusion}

\footnotetext{
${ }^{12}$ Synthèse du forum « Gouvernance des associations et fondations. Intérêts et limites de la bonne gouvernance des associations et des fondations qui font appel à la générosité du public : les grandes lignes du débat », synthèse réalisée par Estelle Hédouin pour le Comité de la Charte, http://www.comitecharte.org/ewb_pages/a/actualite360.php
} 
Les entreprises de l'ESS ont le vent en poupe et ce particulièrement en ces moments de crise et de remise en cause du modèle de l'entreprise capitaliste « traditionnelle ». Ces entreprises sont présentées comme un modèle alternatif qui permettrait « un retour aux principes, à la valorisation de la démocratie et de l'égalité dans les domaines de la production, de la distribution et de l'intermédiation financière " (Singer, 2006, 300). Elles développent un mode entrepreneurial "caractérisé par des règles de gouvernance, de gestion, d'organisation elles-mêmes spécifiques, (dont) découle des formes ou manifestations d'utilité sociale spécifique » (Parodi, 2010, p. 54). Les entreprises de l'ESS doivent donc développer des outils pour s'assurer de leur utilité sociale et du respect des règles de gouvernance. Ces outils ont parfois insuffisants, ou encore en pleine construction, il serait possible à cet égard de s'inspirer des outils mis en œuvre dans le domaine de l'économie capitaliste ou dans celui $\mathrm{du}$ «social business », zone «à cheval » regroupant les entreprises organisées comme celles du secteur marchand mais poursuivant un but social (Hurstel, 2009, p. 17). En effet, les entreprises, qu'elles soient «traditionnelles » ou de l'ESS, partagent de plus en plus des préoccupations identiques quant à leur responsabilité sociale ou la nécessité de mettre en place une bonne gouvernance organisationnelle. Les proclamations de nombreuses entreprises qui se déclarent socialement responsables conduisent celles qui le sont véritablement à mesurer et à légitimer leur action (la mise en place de la norme ISO 26000 sur la responsabilité sociétale des organisations constitue en la matière une avancée significative).

Plusieurs pistes de recherche sont donc ouvertes. Il serait intéressant, pour compléter cette présente étude, d'aller à la rencontre des acteurs de l'ESS afin de recenser de façon plus exhaustive les outils qu'ils mettent en place pour tenir compte des intérêts des parties prenantes. Une recherche comparative pourrait également être menée afin d'étudier la façon dont les entreprises de l'économie capitaliste et celles de l'ESS prennent en considération le concept de RSE et développent des outils afin de mesurer et d'améliorer leurs performances sociales. Cette étude pourrait également essayer de voir si des outils utilisés par les entreprises de l'économie capitaliste pourraient être repris par les entreprises de l'ESS en prenant en considération leurs spécificités (utilité sociale difficilement quantifiable ou alors nécessitant des outils adaptés...).

La question de l'appartenance d'une entreprise à l'ESS pourrait être simplifiée en cas de mise en œuvre de la proposition de la mission parlementaire sur le développement de l'ESS ${ }^{13} \mathrm{~d}^{\prime}$ ' engager une démarche de création de labels qui dépasse la seule approche statutaire pour mieux reconnaître l'appartenance à l'ESS » (propositions 4 et 5). Ce label permettrait de donner aux entreprises de l'ESS une visibilité. Le Mouvement des entrepreneurs sociaux (Mouves) travaille sur ce label (critères d'appartenance, modalités de mise en œuvre.... $)^{14}$ dont le Ministre en charge de l'ESS Benoît Hamont souhaite faire la pierre angulaire de la future loi-cadre sur l'économie sociale et solidaire. Les entreprises de l'ESS semblent donc être sur le point de bénéficier d'une qualification juridique spécifique.

\footnotetext{
${ }^{13}$ Mission présidée par Francis Vercamer et ayant remis son rapport au gouvernement le 28 avril 2010.

${ }^{14} \mathrm{http}: / /$ mouves.org/pg/pages/view/2215/
} 
C. Amblard, (2010), «Intérêt général, utilité publique ou utilité sociale : quel mode de reconnaissance pour le secteur associatif ? », Recma, $\mathrm{n}^{\circ} 315$, p. 21-39.

M. Autès, (2006), «Les acteurs et les référentiels » Les dynamiques de l'économie sociale et solidaire, sous la direction de Jean-Noël Chopart, Guy Neyret et Daniel Rault, La Découverte.

J.-P. Bertrel, (1997), « La position de la doctrine sur l'intérêt social », Droit et patrimoine, avril 1997, p. 42.

M.-P. Blin-Franchomme, (2008), «Entreprises et responsabilité : aperçu de quelques avancées récentes du développement durable dans la vie des affaires... », Lamy Droit des affaires, ${ }^{\circ} 32$, p. 61 67.

M.-P. Blin-Franchomme, (2009), « De quelques éléments de régulation des démarches volontaires en matière de RSE... », Revue Lamy droit des affaires, $\mathrm{n}^{\circ} 40$, p. 71-74.

M. Borgetto, (2006), «L'économie sociale et solidaire: quelle place pour le droit?» in Les dynamiques de l'économie sociale et solidaire, sous la direction de Jean-Noël Chopart, Guy Neyret et Daniel Rault 2006, La Découverte.

S. Castro, (1996), «Le tiers secteur face au droit», in Le secteur sans but lucratif, E. Archambault (dir.), Economica.

Y. Chaput, (2005), «Le monde idéal : les principes de la gouvernance d'entreprise », in Cahiers de droit de l'entreprise, supplément à la Semaine juridique, n ${ }^{\circ} 44-45$ du 3 novembre 2005, p. 25-26.

A. Couret, (2009), « La gouvernance dans les sociétés anonymes, un bouclier pour les dirigeants ?» in Revue Lamy droit des affaires, $\mathrm{n}^{\circ} 41$.

D. De La Garanderie, (2008), « Le contrôle des «parachutes dorés » », D Entretien 2584.

J. Defourny et M. Nyssens, (2011), «Approches européennes et américaines de l'entreprise sociale : une perspective comparative $»$, Recma, $\mathrm{n}^{\circ} 319$, p. 18-35.

J. Delga, (2005), «Propos iconoclastes sur l'éthique d'entreprise : Une analyse juridique de l'éthique d'entreprise au regard de la mondialisation », Revue Lamy Droit des affaires, $\mathrm{n}^{\circ}$ 84, p. 5-9.

I. Desbarats, (2008), « Regard sur un instrument majeur de la gouvernance d'entreprise : quid de la nature juridique des codes éthiques ? », Revue Lamy droit des affaires, $\mathrm{n}^{\circ} 32$, p. 68-70.

A. Euillet, (2002), «L'utilité sociale, une notion dérivée de celle d'intérêt général », Revue de droit sanitaire et social, 30 (2).

Y. Guyon et G. Coquereau, (1984), «Les réformes apportées... par la loi du $1^{\text {er }}$ mars 1984, JCP éd. E, $\mathrm{I}, \mathrm{n}^{\circ} 13426$.

B. Enjolras, (2005), «Economie sociale et solidaire et régimes de gouvernance », Recma, p. 56-69.

B. Enjolras, (2009), «Approche théorique de la gouvernance des organisations non lucratives », Recma, ${ }^{\circ} 314$, p. 63-83.

R. E. Freeman, (1984), Strategic management: a stakeholder approach, Pitman, Boston.

J. Gadrey, (2006), «L'utilité sociale en question : à la recherche de conventions, de critères et de méthodes d'évaluation». Les dynamiques de l'économie sociale et solidaire. La Découverte.

J-P. Gond et S. Mercier, (2006), «La théorie des parties prenantes », in Allouche J. (coord.), Encyclopédie des Ressources Humaines, 2e édition, Vuibert, 2006, p. 917-925.

M. Hély, (2010), «le travail «d'utilité sociale» dans le monde associatif », Revue management \& avenir, $\mathrm{n}^{\circ} 40$, p. 206-217.

M. Hérail, (2002), « Coopérative », Rép. sociétés Dalloz, n 47 et 425.

G. Herry et D. Lenoir (dir.), (2006), «Rapport sur la gouvernance des mutuelles », Assemblée générale de la mutualité française.

D. Hiez et R. Laurent, (2011), «La nouvelle frontière de l'économie sociale et solidaire : l'intérêt général ? », Recma, n 319, p. 36-56.

D. Hurstel, (2009), La Nouvelle Economie sociale. Pour réformer le capitalisme, Odile Jacob.

P. Kaminski, (2009), «Le poids de l'Economie sociale dans le PIB : entre 6 et 7\% ?», XIIème Colloque de l'ADDES.

R. Lafore, (2003), «La mise en forme juridique », in Itçaina X., Lafore R. et Sorbets C. (dir.), Générer et gérer du bien collectif en Aquitaine. Un monde en quête de reconnaissance, CERVL, IEP de Bordeaux.

C. Lapenu et F. Doligez, (2007), «Mesure des performances sociales : les implications pour le secteur de la microfinance », Recma, p. 46-62. 
D. Le Gros et M. Narcy, (2004), « Différentiel de salaire entre secteur sans but lucratif et secteur privé en France », in Solidarités collectives. Famille et solidarités, (Tome 1), Girard D. (dir.), L'Harmattan, p. $215-230$.

D. Lenoir, (2009), «La mutualité face à ses enjeux », Revue de droit sanitaire et social, p. 397.

A. Lienhard, (2008), D, Actu. 2492. Ces recommandations peuvent être consultées en ligne sur le site du MEDEF : www.medef.fr.

A. Lipietz, (2000), L'opportunité d'un nouveau type de société à vocation sociale, (rapport d'étape), cité par Michel Borgetto, p. 236.

C. Malecki, (2009), «L'investissement socialement responsable : quelques problématiques actuelles », Revue Lamy Droit des affaires, $\mathrm{n}^{\circ} 40$.

G. Neyret, (2006), «Mise en perspective des recherches », in Les dynamiques de l'économie sociale et solidaire, sous la direction de Jean-Noël Chopart, Guy Neyret et Daniel Rault 2006, La Découverte.

M. Parodi, (2010), «L'utilité sociale pour éclairer la face cachée de la valeur de l'économie sociale », Recma, $\mathrm{n}^{\circ} 315, \mathrm{p}$ 40-55.

L. Parisot, J.-M. Folz et C. Charrière-Bournazel, « Ne jetons pas l'autorégulation avec l'eau du bain », La Tribune du 16/10/2008, p. 28.

B. Pereira, (2009), "Chartes et codes de conduite : le paradoxe éthique », Revue Management et avenir, $\mathrm{n}^{\circ} 21$, p. 30 .

E. Persais, (2006), «Bilan sociétal : la mise en œuvre du processus RSE au sein du secteur de l'économie sociale », Recma, 2006, p. 14-39.

D. Plantamp, (2005), «La société coopérative d'intérêt collectif et les principes généraux du droit coopératif », RTD Com., p. 465.

J. E. Post, L. E Preston et S. Sachs, (2002), « Managing the Extended Enterprise : the New Stakeholder View», California Management Review, vol. 45, $\mathrm{n}^{\circ}$ 1, fall, p. 6-28.

D. Schmidt, (1995), « De l'intérêt social », Revue Droit Bancaire et Bourse, p. 130.

P. Singer, (2006), «Economie Solidaire (1)», in Laville J.-L., Cattani D., sous la dir., (2006),

Dictionnaire de l'autre économie, Gallimard, Collection Folio Actuel, Paris, p. 290-302.

F.-G. Trébulle, (2004), « La comptabilisation de l'environnement », Droit des sociétés, p. 9.

F.-G. Trébulle, (2009), «Entreprise et développement durable », JCP E n 38, 1867.

M. Waline, (1999), «L'intérêt général », rapport public du Conseil d'Etat, 1999. 